\title{
Data quality of the monoclonal gammopathy of undetermined significance diagnosis in a hospital registry
}

This article was published in the following Dove Press journal:

Clinical Epidemiology

26 August 2013

Number of times this article has been viewed

\author{
Henrik Gregersen' \\ Caroline Brenner Larsen' \\ Anne Haglund' \\ Rikke Mortensen ${ }^{2}$ \\ Niels Frost Andersen ${ }^{3}$ \\ Mette Nørgaard ${ }^{2}$ \\ 'Department of Hematology, \\ Aalborg University Hospital, \\ Aalborg, Denmark; ${ }^{2}$ Department of \\ Clinical Epidemiology, ${ }^{3}$ Department \\ of Hematology, Aarhus University \\ Hospital, Aarhus, Denmark
}

Objective: To estimate the positive predictive value (PPV) and completeness of the monoclonal gammopathy of undetermined significance (MGUS) diagnosis coding in a hospital registry within a population-based health-care setting.

Patients and methods: Through the Danish National Patient Registry (DNPR), we identified 627 patients registered with MGUS in two Danish regions during the period January 2001-February 2011. We reviewed the medical records of all patients registered with MGUS at the Department of Hematology, Aalborg University Hospital, and a sample of patients registered at the other three hematological departments in the two regions. We estimated the PPV of the MGUS diagnosis based on this sample of 327 medical records. We also estimated the completeness of the DNPR by linking data from the DNPR and data from a previously validated MGUS cohort of 791 patients identified through the laboratory system covering North Jutland Region.

Results: The diagnosis of MGUS was confirmed in 231 patients and assessed as probable in an additional 38 patients, corresponding to a PPV of $82.3 \%$ (95\% confidence interval [CI] $78.1 \%-86.4 \%)$. By contrast, $58(17.7 \%)$ of the patients did not definitively meet the diagnostic criteria for MGUS. When we excluded patients registered with malignant monoclonal gammopathy recorded prior to or within the first year after registration of MGUS in the DNPR, the PPV increased to $88.3 \%$ (95\% CI 84.5\%-92.1\%). The DNPR only registered a diagnosis of MGUS in 133 of the 791 MGUS patients identified through the laboratory system, corresponding to a completeness of $16.8 \%$ (95\% CI 14.1\%-19.6\%).

Conclusion: The PPV of the diagnosis coding for MGUS in the DNPR is high and can be further improved by simple data restriction. However, the low completeness raises concern that MGUS patients registered in the hospital system may be highly selected.

Keywords: monoclonal gammopathy of undetermined significance, hospital discharge data, validation, administrative data, ICD-10

\section{Introduction}

Monoclonal gammopathy of undetermined significance (MGUS) is the most common immunoglobulin (Ig) disorder, with an estimated prevalence of $5 \%$ in persons above 70 years of age. ${ }^{1}$ Patients with MGUS are at increased risk of malignant transformation to symptomatic malignant monoclonal gammopathy, most often multiple myeloma and less frequently another malignant lymphoproliferative disorder. The risk of progression to multiple myeloma or related disorders is about $1 \%$ per year. ${ }^{2}$ Recent large population-based cohort studies indicate that MGUS patients also have an increased risk of other clinical events, eg, fractures, infections, and venous and arterial thromboembolism. ${ }^{3-8}$ The clinical impact of these findings is not yet determined, and
Correspondence: Henrik Gregersen Department of Hematology,

Aalborg University Hospital, Box 56I,

Aalborg DK-9000, Denmark

Tel +4599326326

Fax +4599326108

Email henrik.gregersen@rn.dk 
it is unknown to what degree they are an innate feature of MGUS or might be affected by comorbidity.

Administrative data are an important source of information in epidemiological studies, and provide a cost-efficient way of studying large cohorts, often in a regional or nationwide population-based setting. ${ }^{9}$ In MGUS, the medical registries might also have an important role in estimating the risk of various outcomes as these patients are followed in different settings, eg, outside hematological departments, and the risk of the outcomes is in general low. Consequently, there is a need for including a large number of MGUS patients with long-term follow-up to estimate the risk. However, a prerequisite for this type of study is high-quality data on MGUS patients.

No previous studies have formally addressed the validity and completeness of the MGUS diagnosis in a hospital registry. We therefore conducted a study in two Danish regions to estimate the positive predictive value (PPV) and completeness of the MGUS diagnosis in the Danish National Patient Registry (DNPR).

\section{Design and methods}

We conducted the study in North Jutland Region and Central Jutland Region in Denmark, which have 1.8 million inhabitants (approximately 33\% of the total Danish population). Every citizen in Denmark is assigned a personal identification number at birth, which allows linkage between demographic and medical registries. ${ }^{10}$ Since 1977 , the DNPR has routinely collected nationwide data on all nonpsychiatric hospitalizations, and since 1995, visits to emergency departments and outpatient clinics have also been included. ${ }^{11}$ The recorded data include dates of admission and discharge, patient type (ie, outpatient or inpatient), primary discharge diagnosis, reflecting the main reason for hospitalization or outpatient visit, and up to 20 secondary discharge diagnoses. The diagnoses have been coded according to the International Classification of Diseases, 10th revision (ICD-10) since 1994.

\section{Subjects coded with MGUS}

We identified 627 persons in North Jutland Region and Central Jutland Region registered in the DNPR with the MGUS ICD-10 code D47.2 in the period January 2001February 2011.

\section{Validation of the MGUS ICD-I0 code}

We reviewed the medical records of all 132 subjects with MGUS registered at the Department of Hematology, Aalborg University Hospital in North Jutland Region, and a sample of 174 patients registered at the hematological departments in Central Jutland Region at Aarhus University Hospital and the two regional hospitals (Viborg and Holstebro). In addition, we reviewed 21 available medical records on patients registered at other departments in North Jutland Region. In total, 327 of the 627 medical records were reviewed, which was done by three of the authors (HG, CBL, AH).

MGUS was defined according to the 2003 criteria for classification of monoclonal gammopathies from the International Myeloma Working Group. ${ }^{12}$ According to these criteria, the MGUS diagnosis requires a concentration of the serum M-component of less than $30 \mathrm{~g} / \mathrm{L}$, percentage of monoclonal plasma cells in the bone marrow less than $10 \%$, and no sign of organ or tissue impairment related to myeloma, eg, hypercalcemia or lytic bone lesions. Patients without skeletal survey were classified as MGUS provided that bone pain was not described in the medical files. A subgroup of patients was classified as probable MGUS for various reasons, eg, patients with renal failure or hypercalcemia who had plausible other causes for these abnormalities or lytic bone lesions caused by other cancers.

\section{Subjects with MGUS in North Jutland Region detected via data from the Department of Clinical Biochemistry}

Since 1978, the Department of Clinical Biochemistry at Aalborg University Hospital has maintained a registry of all patients in North Jutland Region with a serum M-component identified by electrophoresis. Patients with an M-component were classified as having MGUS if they were not registered in the DNPR with malignant monoclonal gammopathy prior to or within the year following detection of the M-component. In order to exclude cases of asymptomatic multiple myeloma, we excluded patients if their levels of $\operatorname{IgA}, \operatorname{IgG}$, and $\operatorname{IgM}$ were $30 \mathrm{~g} / \mathrm{L}$ or higher. Identification and verification of this MGUS cohort has previously been described in detail. ${ }^{7,13}$ In the period January 2001-February 2011, we identified 791 patients with MGUS using this algorithm. These data were linked to the DNPR to estimate the number registered with MGUS.

\section{Comorbidity}

Comorbidity was classified according to the 19 diseases included in the Charlson Comorbidity Index (CCI). ${ }^{14}$ We computed a CCI score for each MGUS patient based on all previous ICD codes in the DNPR since 1977. Weights were assigned to defined categories of comorbid diseases, and the CCI score was the sum of these weights. Four levels of 
comorbidity were defined: 0 (low) for individuals with no recorded underlying diseases included in the CCI, 1 (moderate low), 2 (moderate high), and $\geq 3$ (high).

\section{Statistical analysis}

The PPV of the diagnosis of MGUS in the DNPR was calculated as a proportion, with the numerator containing the number of patients with a confirmed diagnosis of MGUS and the denominator containing the number of reviewed medical files. We estimated PPVs separately for the different subgroups of MGUS patients, eg, in different types of M-components. The completeness of MGUS registration in the DNPR was estimated as the percentage of patients with MGUS based on the data from the Department of Clinical Biochemistry, Aalborg University Hospital, who were registered with MGUS in the DNPR. We used the SAS statistical software package, version 9.2 (SAS Institute, Cary, NC, USA) for the statistical analyses. The study was approved by the Danish Data Protection Agency (200858-0028) and the Danish Health and Medicines Authority (3-3013-54/1/HKR).

\section{Results}

At registration of MGUS in the DNPR, the median age of the 327 patients was 71.0 years (range 34.6-91.7 years), and 166 (51\%) were men. In 219 patients, the diagnosis was registered at the two university hospitals, in 87 patients at the two large regional hospitals, and in 21 patients at smaller hospitals in North Jutland Region. In 50 cases (15.3\%), the serum M-component was IgA, compared to IgG in 185 cases (56.6\%), $\operatorname{IgM}$ in 67 cases $(20.5 \%)$, biclonal in seven cases $(2.1 \%)$, exclusively light chain in four cases $(1.2 \%)$, and in one case $(0.3 \%)$ the type of M-component was not reported. In 13 patients (4\%) a serum M-component was not detected, but two of these patients had M-component identified in the urine.

\section{Validation of the MGUS diagnosis code}

The diagnostic workup involved bone marrow examination in $300(91.7 \%)$ and skeletal survey in $223(68.2 \%)$ patients. In addition, 15 patients had other types of imaging performed, most often computed tomography scans, increasing the number of patients investigated with radiological techniques to $238(72.8 \%)$. Measurement of Ig levels and standard biochemical and hematological parameters was performed consistently. However, in 14 patients, all from one of the regional hospitals, we were not able to retrieve these results. Electrophoresis on urine was performed in 195 (59.6\%) of the patients.
The review of the medical records confirmed the MGUS diagnosis in 231 patients, and assessed MGUS as probable in an additional 38 patients, corresponding to a PPV of $82.3 \%$ (95\% confidence interval [CI] 78.1\%-86.4\%). The PPVs in different subgroups of MGUS patients are shown in Table 1. The criteria for MGUS were not fulfilled in 58 patients $(17.7 \%)$. In $16(27.6 \%)$ of these 58 patients, the diagnosis was asymptomatic myeloma with a median plasma cell

Table I Positive predictive value (PPV) of a diagnosis of monoclonal gammopathy of undetermined significance in 327 patients registered in the Danish National Patient Registry

\begin{tabular}{|c|c|c|c|}
\hline & $\begin{array}{l}\text { Correct } \\
\text { registration }\end{array}$ & $\begin{array}{l}\text { Incorrect } \\
\text { registration }\end{array}$ & $\begin{array}{l}\text { PPV } \\
\text { (95\% confidence } \\
\text { interval) }\end{array}$ \\
\hline \multicolumn{4}{|l|}{ Sex } \\
\hline Women & $|3|$ & 30 & $\begin{array}{l}81.4 \% \\
(75.4 \%-87.4 \%)\end{array}$ \\
\hline Men & 138 & 28 & $\begin{array}{l}83.1 \% \\
(77.4 \%-88.8 \%)\end{array}$ \\
\hline \multicolumn{4}{|l|}{ Age } \\
\hline$>70$ years & 128 & 36 & $\begin{array}{l}78.0 \% \\
(71.7 \%-84.9 \%)\end{array}$ \\
\hline$\leq 70$ years & $|4|$ & 22 & $\begin{array}{l}86.5 \% \\
(81.3 \%-91.7 \%)\end{array}$ \\
\hline \multicolumn{4}{|l|}{ Registration year } \\
\hline $200 \mathrm{I}-2005$ & 106 & 25 & $\begin{array}{l}80.9 \% \\
(74.2 \%-87.7 \%)\end{array}$ \\
\hline $2006-2011$ & 163 & 33 & $\begin{array}{l}83.2 \% \\
(77.2 \%-88.4 \%)\end{array}$ \\
\hline \multicolumn{4}{|l|}{ M-component type } \\
\hline Free light chain & 2 & 2 & $\begin{array}{l}50.0 \% \\
(1.0 \%-99.0 \%)\end{array}$ \\
\hline $\lg A$ & 39 & 11 & $\begin{array}{l}78.0 \% \\
(66.5 \%-89.5 \%)\end{array}$ \\
\hline $\lg G$ & 159 & 26 & $\begin{array}{l}85.9 \% \\
(80.9 \%-91.0 \%)\end{array}$ \\
\hline $\lg M$ & 61 & 6 & $\begin{array}{l}91.0 \% \\
(84.2 \%-97.9 \%)\end{array}$ \\
\hline Biclonal & 6 & I & $\begin{array}{l}85.7 \% \\
(59.8 \%-100 \%)\end{array}$ \\
\hline \multicolumn{4}{|l|}{ Comorbidity score } \\
\hline 0 & 121 & 14 & $\begin{array}{l}89.6 \% \\
(84.5 \%-94.8 \%)\end{array}$ \\
\hline 1 & 55 & 14 & $\begin{array}{l}79.7 \% \\
(70.2 \%-89.2 \%)\end{array}$ \\
\hline 2 & 48 & 13 & $\begin{array}{l}78.7 \% \\
(68.4 \%-89.0 \%)\end{array}$ \\
\hline$\geq 3$ & 49 & 13 & $\begin{array}{l}79.0 \% \\
(68.9 \%-89.2 \%)\end{array}$ \\
\hline \multicolumn{4}{|l|}{ Hospital } \\
\hline University hospitals & 183 & 36 & $\begin{array}{l}83.6 \% \\
(78.7 \%-88.5 \%)\end{array}$ \\
\hline Regional hospitals & 69 & 18 & $\begin{array}{l}79.3 \% \\
(70.8 \%-87.8 \%)\end{array}$ \\
\hline Other hospitals & 17 & 4 & $\begin{array}{l}81.0 \% \\
(64.2 \%-97.8 \%)\end{array}$ \\
\hline
\end{tabular}

Abbreviation: Ig, immunoglobulin. 
percentage of $11.5 \%$ (range $10 \%-30 \%$ ), whereas symptomatic myeloma was found in 23 (39.7\%) of the patients (Table 2). Eight patients $(13.8 \%)$ had another malignant monoclonal gammopathy. By contrast, no sign of either MGUS or malignant monoclonal gammopathy was found in eleven patients (18.9\%). None of the 58 patients was misclassified due to high concentration of the M-component ( $>30 \mathrm{~g} / \mathrm{L})$.

In the DNPR, 54 of the patients were registered with malignant monoclonal gammopathy, either prior to the MGUS diagnosis or within the first year after registration of MGUS. The review of medical records had classified 28 of these patients as MGUS and 26 as not fulfilling the MGUS criteria. When we excluded the 54 patients from the analysis, the PPV increased to $88.3 \%$ (95\% CI 84.5\%-92.1\%).

\section{Completeness of MGUS registration}

During the study period, 791 individuals were identified as having MGUS based on data from the Department of Clinical Biochemistry, Aalborg University Hospital. The median age was 71.7 years, range 22.9-97.2 years, and 457 (57.8\%) were men. After first-time detection of an M-component, 133 patients were subsequently registered with MGUS in the DNPR, corresponding to a completeness of $16.8 \%$ (95\% CI 14.1\%-19.6\%). The median age in this cohort of registered patients was 68.9 years, range 35.9-86.8 years, and $70(52.6 \%)$ were men. The median time from detection of an M-component to registration of MGUS in the DNPR was 46 days (range $0-8.3$ years).

\section{Discussion}

We found that the PPV of the MGUS diagnosis in the DNPR was $82.3 \%$, and that it could be improved to $88.3 \%$ by simple data restriction. Conversely, the completeness of MGUS diagnoses in this national hospital registry was very low. To our knowledge, this is the first study to address the data quality of the ICD-10 code for MGUS in a patient registry.

The PPV of the registration of MGUS in our study was in particular affected by misclassification of myeloma patients as MGUS. Approximately half of the misclassified cases had

Table 2 Diagnoses in the 58 patients who were incorrectly registered with a diagnosis of monoclonal gammopathy of undetermined significance

\begin{tabular}{ll}
\hline Asymptomatic myeloma & $16(27.6 \%)$ \\
Symptomatic myeloma & $23(39.7 \%)$ \\
Malignant lymphoma & $7(12.1 \%)$ \\
Chronic lymphocytic leukemia & $1(1.7 \%)$ \\
Transient M-component & $1(1.7 \%)$ \\
No serum M-component detected & $10(17.2 \%)$ \\
\hline
\end{tabular}

asymptomatic myeloma, which implies an increased risk of development of symptomatic multiple myeloma compared to MGUS. ${ }^{15}$ The impact of this misclassification on the risk of other outcomes is unknown, but it is noteworthy that most of the misclassified patients with asymptomatic multiple myeloma had a percentage of plasma cells in the bone marrow just above the limit of $10 \%$ that distinguish the two conditions. This misclassification in the continuum of monoclonal gammopathies might therefore not seriously affect the evaluation of particular outcomes, eg, risk of infections. By contrast, the misclassification of patients with symptomatic myeloma as MGUS will bias the estimate of most outcomes, eg, the risk of fractures and infections.

The International Myeloma Workshop (IMW) criteria provide a strong and precise tool for classification of the monoclonal gammopathies, and had been used in Denmark for the entire study period with the exception of the first 2 years. ${ }^{12}$ However, our study clearly demonstrates that correct diagnosis of monoclonal gammopathies might prove difficult. The diagnostic process of MGUS is complex, as the diagnosis is based on a combination of results of bone marrow examination, biochemical analysis, and radiological tests. Our study also demonstrated that the diagnostic workup was not complete in all patients, including some patients at hematological departments, which most likely will affect the validity of the diagnosis. Although bone marrow findings are a part of the diagnostic criteria for MGUS, there is no consensus regarding the requirement for bone marrow aspirate and biopsy for patients who have been diagnosed with a monoclonal gammopathy. ${ }^{16,17}$ Conversely, some authors have argued for consistent use of bone survey in patients with assumed MGUS, in order to identify possible lytic lesions and fractures that may indicate symptomatic treatment-demanding multiple myeloma. ${ }^{18}$ In our study, radiological investigations were performed in approximately two-thirds of the patients coded with MGUS.

IgM MGUS is not covered by the IMW criteria, and the distinction between malignant lymphoma and IgM MGUS is based on examination of the bone marrow or other tissue samples and exclusion of other signs of malignant lymphoproliferative disease. In most cases, the differential diagnosis is between IgM MGUS and asymptomatic Waldenström's macroglobulinemia (lymphoplasmacytic lymphoma). ${ }^{19}$ Our study did not permit an evaluation of the pathological investigation, but PPV of the IgM MGUS diagnosis code was in general high.

Only $17 \%$ of the patients identified with an M-component at the Department of Clinical Biochemistry and without a concomitant diagnosis of malignant monoclonal gammopathy in 
the DNPR had an MGUS diagnosis registered in the DNPR. This proportion is surprisingly low. Our study design did not allow evaluation of whether the serum M-component was detected by general practitioners without the patient being referred for diagnostic workup at a hospital, or alternatively that the M-component was detected at a hospital without subsequent registration of MGUS. However, our findings emphasize an important issue in the diagnostic process, namely that detection of a serum M-component in daily clinical practice does not necessarily lead to a diagnostic workup and to registration of MGUS. Due to the design of our study, we are not able to evaluate whether these unregistered MGUS patients in fact were perceived and managed as MGUS patients in clinical practice. The low completeness clearly demonstrates that hospital register-based diagnoses of MGUS are not suitable for estimating the prevalence of MGUS, and indicates that MGUS patients coded in these registries are likely to be highly selected. Serum protein electrophoresis is usually performed on selected groups of patients, often with symptomatology indicative of a malignant monoclonal gammopathy or other serious diseases. Furthermore, the referral of patients for medical workup will presumably be affected by the symptoms of the patients, which might lead to imbalance of prognostic factors in patients registered with MGUS compared to patients who remain unregistered.

\section{Conclusion}

In conclusion, we found a high PPV of the MGUS diagnosis in the DNPR, which supports previous published registerbased studies on MGUS and confirms that such administrative data constitute a strong tool for future epidemiological research in MGUS. However, the completeness in the DNPR is low, indicating that only a small fraction of patients with detected M-components get an MGUS diagnosis in the hospital setting. It emphasizes the risk of selection and underlines the need for MGUS studies based on MGUS patients identified from screening programs or cross-sectional populations.

\section{Acknowledgments}

The study received financial support from the Obel Family Foundation.

\section{Disclosure}

The authors report no conflicts of interest in this work.

\section{References}

1. Kyle RA, Therneau TM, Rajkumar SV, et al. Prevalence of monoclonal gammopathy of undetermined significance. NEngl JMed. 2006;354(13): 1362-1369.

2. Rajkumar SV, Kyle RA, Therneau TM, et al. Serum free light chain ratio is an independent risk factor for progression in monoclonal gammopathy of undetermined significance. Blood. 2005;106(3):812-817.

3. Gregersen H, Madsen KM, Sørensen HT, Schønheyder HC, Ibsen JS, Dahlerup JF. The risk of bacteremia in patients with monoclonal gammopathy of undetermined significance. Eur J Haematol. 1998;61(2): $140-144$.

4. Kristinsson SY, Tang M, Pfeiffer RM, et al. Monoclonal gammopathy of undetermined significance and risk of skeletal fractures: a populationbased study. Blood. 2010;116(15):2651-2655

5. Kristinsson SY, Pfeiffer RM, Björkholm M, et al. Arterial and venous thrombosis in monoclonal gammopathy of undetermined significance and multiple myeloma: a population-based study. Blood. 2010;115(24): 4991-4998.

6. Gregersen H, Nørgaard M, Severinsen MT, Engebjerg MC, Jensen P, Sørensen HT. Monoclonal gammopathy of undetermined significance and risk of venous thromboembolism. Eur J Haematol. 2011;86(2): $129-134$.

7. Gregersen H, Jensen P, Gislum M, Jørgensen B, Sørensen HT, Nørgaard M. Fracture risk in patients with monoclonal gammopathy of undetermined significance. Br J Haematol. 2006;135(1):62-67.

8. Kristinsson SY, Tang M, Pfeiffer RM, et al. Monoclonal gammopathy of undetermined significance and risk of infections: a population-based study. Haematologica. 2012;97(6):854-858.

9. Sorensen HT, Sabroe S, Olsen J. A framework for evaluation of secondary data sources for epidemiological research. Int J Epidemiol. 1996;25(2):435-442.

10. Frank L. Epidemiology. When an entire country is a cohort. Science. 2000;287(5462):2398-2399.

11. Andersen TF, Madsen M, Jørgensen J, Mellemkjaer L, Olsen JH. The Danish National Hospital Register. A valuable source of data for modern health science. Dan Med Bull. 1999;46(3):263-268.

12. International Myeloma Working Group. Criteria for the classification of monoclonal gammopathies, multiple myeloma and related disorders: a report of the International Myeloma Working Group. Br J Haematol. 2003;121(5):749-757.

13. Gregersen H, Mellemkjaer L, Ibsen JS, et al. Cancer risk in patients with monoclonal gammopathy of undetermined significance. Am J Hematol. 2000;63(1):1-6.

14. Charlson ME, Pompei P, Ales KL, MacKenzie CR. A new method of classifying prognostic comorbidity in longitudinal studies: development and validation. J Chronic Dis. 1987;40(5):373-383.

15. Rago A, Grammatico S, Za T, et al. Prognostic factors associated with progression of smoldering multiple myeloma to symptomatic form. Cancer. 2012;118(22):5544-5549.

16. Berenson JR, Anderson KC, Audell RA, et al. Monoclonal gammopathy of undetermined significance: a consensus statement. Br J Haematol. 2010;150(1):28-38.

17. Elis A, Radnay J, Shapiro H, Itzhaky D, Manor Y, Lishner M. Should bone marrow examination be routinely performed for the diagnosis of monoclonal gammopathy of undetermined significance? Isr Med Assoc J. 2006;8(12):840-842.

18. Berenson JR, Yellin O. Monoclonal gammopathy of undetermined significance: why identification of these patients and assessment of their skeletons is important. Clin Lymphoma Myeloma. 2009;9(4): 311-315.

19. Ghobrial IM. Are you sure this is Waldenstrom macroglobulinemia? Hematology Am Soc Hematol Educ Program. 2012;2012:586-594. 


\section{Publish your work in this journal}

Clinical Epidemiology is an international, peer-reviewed, open access journal focusing on disease and drug epidemiology, identification of risk factors and screening procedures to develop optimal preventative initiatives and programs. Specific topics include: diagnosis, prognosis, treatment, screening, prevention, risk factor modification, systematic

Submit your manuscript here: http://www.dovepress.com/clinical-epidemiology-journal reviews, risk \& safety of medical interventions, epidemiology \& biostatical methods, evaluation of guidelines, translational medicine, health policies \& economic evaluations. The manuscript management system is completely online and includes a very quick and fair peer-review system, which is all easy to use. 